\title{
Using structured light three- dimensional surface scanning on living individuals: key considerations and best practice for forensic medicine
}

\begin{abstract}
Non-contact three-dimensional (3D) surface scanning methods have been applied to forensic medicine to record injuries and to mitigate ordinary photography shortcoming. However, there are no literature concerning practical guidance for 3D surface scanning of live victims. This paper aimed to investigate key 3D scanning issues of the live body to develop a series of scanning principles for future use on injured victims. The Pico Scan 3D surface scanner was used on live test subjects. The work focused on analysing the following concerns: 1) an appropriate 3D scanning technique to scan different body areas, 2) the ideal number of scans, 3) scanning approaches to access various areas of the body and 4) elimination of environmental background noise in the acquired data. Results showed that scanning only a required surface of the body area in the stable manner was more efficient when compared to complete $360^{\circ}$-scanning; therefore, it used as a standard 3D scanning technique. More than three scans were sufficient when trying to obtain an optimal wireframe mode presentation of the result. Three different approaches were suggested to provide access to the various areas of the body. Undertaking scanning using a black background eliminated the background noise. The work demonstrated that the scanner will be promising to reconstruct injuries from different body areas, although the 3D scanning of the live subjects faced some challenges.
\end{abstract}

\section{Keywords}

Structured light 3D scanning; live body 3D scanning guidance; forensic wound documentation. 


\section{Introduction}

Although the photography plays an important role in preserving the forensic evidence, ${ }^{1}$ it cannot record the complexity of injuries in depth because these images are flat and only having two dimensions ( $x$ and $y$ ). This means the three-dimensional (3D) injuries are reduced to two-dimensional level, ${ }^{1-4}$ the 3D geometry of the injury, the real intrinsic information and the life size of the injury all are lost. Moreover, the digital photography can produce two dimensional (2D) images with photographic distortion, ${ }^{5,6}$ affected by any kind of noise, ${ }^{7}$ and simply influenced by some factors, such as camera position, angle of acquisition and lighting condition. ${ }^{4}$ These shortcomings have an impact on the accuracy. Therefore, the 2D documents of forensic wound are logically insufficient and less than optimal for wound interpretation at the medico-legal centre and for wound presentation at the court-room. These deficiencies underline why there were recommendations to record forensic injuries using non-contact 3D surface acquisition methods. ${ }^{3,8-10}$

Non-contact 3D surface scanning methods are used to compute 3D coordinates $(x, y, z)$ of object's surface points ${ }^{11}$ and represent the $3 \mathrm{D}$ surface geometry without touching the object. They include two different scanning techniques: passive and active. Passive scanning methods rely on taking series of photographs recording from different angles of the object surface and finding correspondences between the different photos using a photogrammetric software, ${ }^{12}$ i.e., they are photos-based measurement techniques. Active scanning methods reconstruct the surface object by projecting kind of light to the object's surface such as a laser beam or a light pattern. ${ }^{13}$

The active 3D surface scanning techniques can be used as powerful methods to document forensic injuries 3,9 because the 3D outcomes provide a higher level of accuracy and resolution. 3,8,14 They are not new technology, they have been applied for several purposes including, for instance, plastic surgery, facial mapping, recording traffic accident at a crash site, ${ }^{11}$ in addition to the heritage ${ }^{15}$ and archaeological applications. ${ }^{16}$ The active 3D surface scanning method, Streifenlichttopometrie, was introduced to the forensic medicine in 1998 in Germany, ${ }^{14}$ and ATOS structured light 3D scanners have been applied in Switzerland since 2003 ${ }^{3,17,18}$; however, ATOS scanners involve more conditions to produce high resolution results. ${ }^{19}$ Within forensic medicine, active 3D surface scanning technology has limited use due to the cost and the manipulation of the victim required. Thus, it is not completely integrated into the routine forensic work everywhere, even though the method has a potential role in reconstructing the injuries which would have a great significance in the future. ${ }^{9,18}$

Although alternatives passive 3D technology exists, examples such as photogrammetry are not superior in recording forensic injuries since it cannot obtain the same level of the accuracy and points density when compared to the active 3D scanners. ${ }^{4,8,20}$ It is a passive method, does not project coded light; thus, the finding correspondence between photos is more difficult. ${ }^{21}$ The lighting condition may have also an impact on photos matching. ${ }^{4,21}$ Moreover, 3D passive technology is affected by the 
motion and breathing process of a live subject, especially when a single camera photogrammetry is used for full body surface scanning. These impacts produce a set of photos that fails to generate an adequate 3D model. ${ }^{19}$ Thus, obtaining a small number of photos in short time is recommended ${ }^{22,23}$; however, these solutions will add extra deficiency to the quality of the 3D final model. Using a multiple camera photogrammetry to record injuries such as $\mathrm{DI} \mathrm{D}^{10}$ is not practical; in addition to the cost and space requirement. Hairy and wet areas of the body are difficult to record perfectly with passive 3D methods. ${ }^{19,21,22}$ Despite recent advances of 3D passive methods, they are not the perfect choice to reconstruct injuries in live victims.

Laser and structured light scanners are two types of active 3D surface scanning. The structured light scanners are actively used in many applications. ${ }^{24-27}$ Their popularity is underlined by the accuracy of 3D surface geometry coupled with rapid acquisition speeds. ${ }^{26}$ Although all 3D noncontact measurement methods prefer an object to be static, moving objects can be scanned by the 3D structured light technique as it is able to measure almost the entire field of view at once instead of scanning one point. ${ }^{28}$ Coded structured light methods are able to cope with the correspondence problem of the passive 3D technology by giving specific code-words to every point on the object surface. ${ }^{12,24,25,29}$ These coding strategies play an important role in providing accurate 3D results. Also, structured light methods have no safety issues and risk-free to the subjects. These advantages will make it a scanner of choice in recording injuries of the live victims. In contrast, laser scanners are clearly challenged by motion artefacts, ${ }^{23}$ they require objects to be motionless for a longer period. The laser scanners emit a laser beam to the object's surface and moves slowly across the surface then reflects to a sensor, ${ }^{19}$ during the measurement time any movement will lead to distortion of collected image points. ${ }^{30}$ Also, there is a concern about the laser effect on sensitive areas of the body such as the retina. Although there are laser systems designed for human scanning, it is better to avoid this scanner type with human body. Furthermore, 3D laser scanning is hardly digitising dark surfaces as the laser absorbed by the dark color ${ }^{31}$; in addition, it has no automatic reconstruction of the colour texture information into the $3 \mathrm{D}$ mesh. ${ }^{9}$

Although the 3D structured light surface digitization is the method of choice for living individuals, there are no written standard procedure for live victim's 3D scanning. Therefore, the primary objective of this paper involved using the 3D structured light scanner to investigate the 3D scanning issues of the living body for setting up a series of guidelines for future use on injured live subjects. The study centered on determining the appropriate 3D scanning technique to reconstruct a body area, deciding the ideal number of scans and eliminating the environmental background noise in the acquired data and this must be achieved by suggesting applicable approaches to access various areas of the live body. 


\section{Methods}

The Pico Scan 3D scanner was selected to conduct this work. The Pico Scan system is 3D structured light scanner consisting of a Pico LCD projector and a canon EOS 1100D camera. The projector has a resolution of $800 \times 600$ pixels and the camera has a resolution of $968 \times 6448$ bits. The projector projects coded structured light on a measurement surface where the camera captures the images of the illuminated surface. It is well known that the reconstruction of a 3D shape by the structured light scanning is estimated from the deformation of the projected light patterns by the geometric shape of the surface. ${ }^{11,12,18,19,24,28,29}$ The 3D computation of surface points by the Pico scan is based on a Phase Measuring Profilometry (PMP) principle. The PMP principle is one of the most robust and precise method among all 3D structured-light methods. The major benefits of PMP are high accuracy of 3D data measurement, ${ }^{20,25}$ accurate sub-pixel correspondence between the projector and the camera, ${ }^{20}$ high spatial resolution result ${ }^{20,32}$ and robustness to obtain color texture. ${ }^{25}$ In addition to high speed 3D measurement. ${ }^{20,25,33}$ The Pico Scan 3D scanner's results have a point to point distance of $0.16 \mathrm{~mm}$ and a point accuracy of $0.1 \mathrm{~mm}$.

The scanning process was conducted in two main phases: scanning phase followed by post processing phase. The scanning phase was conducted by a Pico Scan 3 software, began with data acquisition. The data acquisition involved illuminating the scanned view with structured light stripes and the camera recorded 14 images of the illuminated surface in addition to one color textured mapping image in each scan (Fig. 1). The acquisition speed was around 18-20 s for each separate scan. The data acquisition followed by data processing and reconstruction of 3D raw data (3D point cloud).

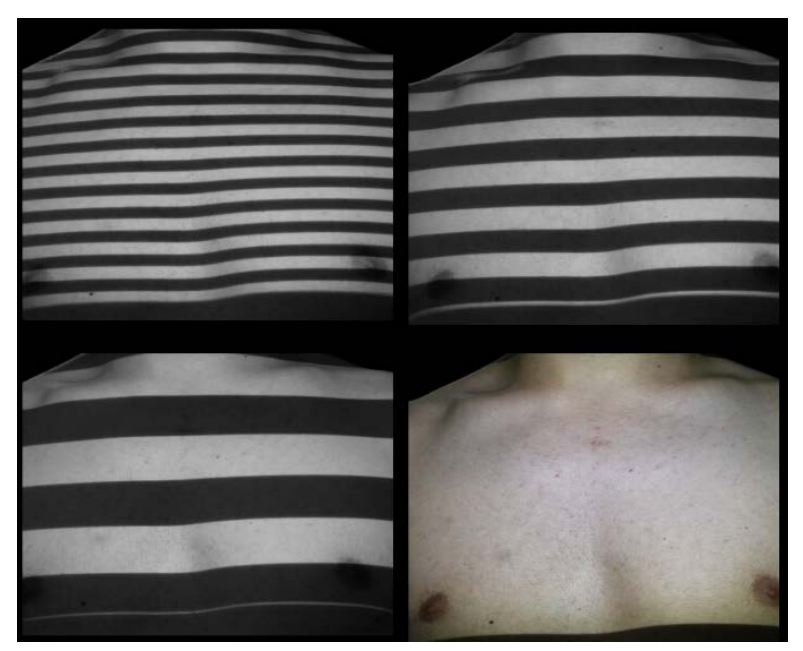

Fig. 1. Some of acquired images with structured light, plus one colour textured mapping image.

In the post processing phase all 3D reconstructed raw data were imported to a separate software program called Mephisto process. This was used for manual noise cleaning of the all data, integration of the all data with the main data, alignment and final processing of the all data. All input data were 
combined into a single 3D data, final 3D model, which consisting of recognizable geometry and color textured information. Exportation of the final 3D image was done in 'Ply' file format which supports vertex color, normals, UV's, points and faces. The final 3D image in Ply file format was presented in the textured and wireframe mode by a 3D MeshLab software for subjective visual assessment. Fig. 2. Summaries the scanning process of the Pico Scan 3D scanner.

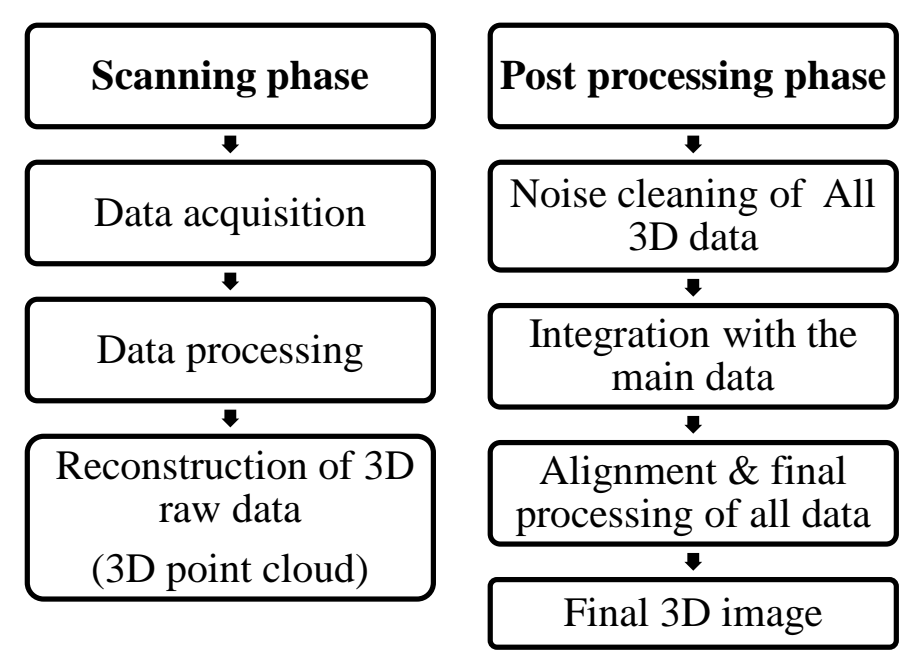

Fig.2. Workflow of scanning process using the Pico Scan 3D Scanner.

In order to have accurate scanning results, the scanning process must be preceded by a geometric calibration procedure. It is the key step which ensures the Pico Scan 3D scanner can attain accurate absolute measurement values. The procedure was achieved with a standard calibration board size, 21x15 squares $(25 \times 18 \mathrm{~cm})$. However, Calibration board can be enlarged or reduced based on the object size. The calibration result was excellent when the values were from 0.2 to 0.5 . Although the calibration procedure guaranteed the accuracy of the results, number of scanning criteria were suggested and followed to raise the efficiency of the results, these criteria include:

1-The 3D reconstructed raw data should be free from environmental background noise and distorted points which generated from the scanned area and caused by motion.

2-The points of the 3D reconstructed raw data should be manipulated as a single cohesive entity in the Mephisto process software.

3-All 3D reconstructed raw data should have the same degree of distributed scanner light and the same degree of the color textured information.

4-All 3D reconstructed raw data should have clear anatomical features of the scanned area.

If the 3D raw data fulfilled these criteria, the data processed to generate the final 3D image.

Volunteers read a research participant information sheet, which contained information about the work, the scanning procedure, the possible risk, confidentiality and right of withdrawal. Informed 
consent was also obtained from all individual participants. Removing jewelry, watch, clothing and other items was required before scanning. The scanning process took place indoor and any source of light was eliminated. The structured light illuminated a localized bare body area corresponding to the calibration board size. The minimum and maximum scanning distance were around 100 and $700 \mathrm{~mm}$ respectively. When the scanning process was completed, the data and images were stored on a password protected university computer and all data were anonymized. The basic 3D scanning issues were investigated on bare different body areas of live test subjects, results analyzed and discussed separately as following:

\section{1-The appropriate 3D scanning technique to reconstruct a body area:}

The technique used for 3D surface scanning of the body area was the primary issue, a decision had to be made whether the area was reconstructed using $360^{\circ}$ scanning, complete scanning over the entire area, or scanning only one surface, desired surface, in the stable manner. The previous works ${ }^{1,5,9,10,22,34}$ reconstructed injuries by scanning only the injured surface; however, in order to decide the scanning technique in this work, a foot was scanned in two ways; i.e., $360^{\circ}$ scanning and scanning only one surface in the stable manner. The scanning process in both methods were undertaken with the same experimental conditions and with the same scanner parameters. Fixing an object and moving the scanner around is not practical when scanning an area of live body; thus, the $360^{\circ}$-foot model was acquired by movement of a volunteer in the constant circular manner from one view to another. The outcome of $360^{\circ}$-foot scanning was multiple 3D raw data. The 3D data which were then integrated and processed together after noise cleaning. A final 3D model based on $360^{\circ}$ foot scanning was visualized in the wireframe mode of the MeshLab software however, overlapping shadow impaired the quality of the final image. When scanning one surface in the unchanging manner, limited data were produced which was desirable. However, using this method also mitigated the unwanted shadow produced in the previous technique. The final 3D image was of good quality and provided clear visualization.

The different areas of the upper limb were difficult to access by the scanner when attempting to scan them in $360^{\circ}$; therefore, the wrist joint was scanned from at least two different views; anterior and anterolateral surface. Also, the area was scanned in the one view, unchanging manner. The final 3D model resulting from scanning the anterior and antero-lateral surfaces of the wrist joint had poor quality due to the overlapping artefact (Fig. 3), whereas the final image generated from scanning only one surface in the stable manner was free from artifact and had clear visualization (Fig. 4). 


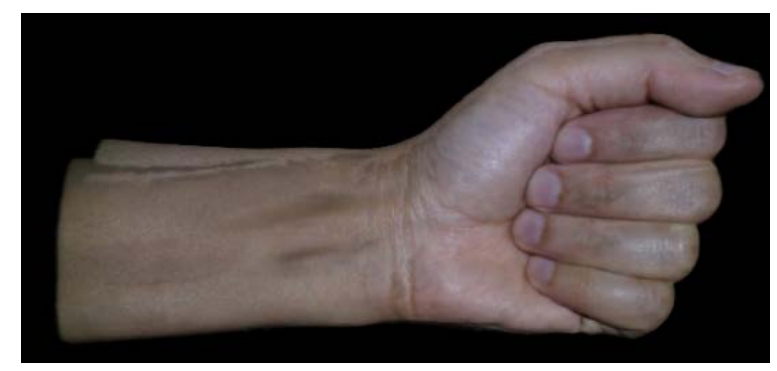

Fig. 3. Final 3D model of the wrist joint with overlapping artefact.

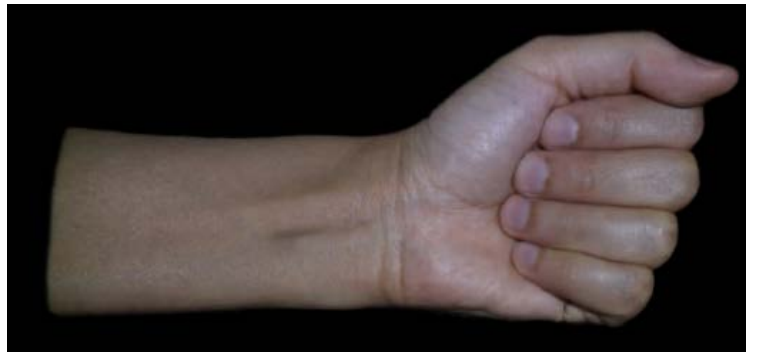

Fig. 4. Final 3D model of the wrist joint free from artefact.

The poor quality of the final 3D model was explained by physiological artifact resulting from muscle activity. When the scanned area was moved from one view to another, the physiology of the movement influenced the result and caused artefact. While scanning a view in the stable manner produced good result free from artefact. Movement from one view to another requires muscle contraction and relaxation and this causes change in the muscle contours (Fig. 5). Therefore, the calculation of 3D coordinates of some previously measured points in the first view will not be in the same 3D position in the new view resulting in some points lose their alignment and overlapping 
shadow artefact. Conversely, scanning a view in the stable manner produced good results free from artefact.

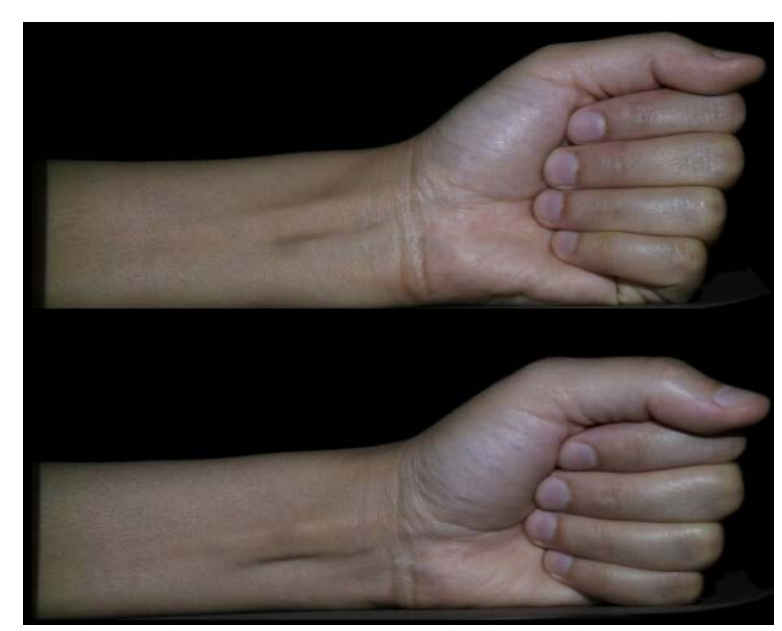

Fig. 5. The bottom image shows the modification of the muscle contour in Thenar eminence, Tendon of Flexor carpi radialis muscle and Tendon of Palmaris longus when the area was moved from the anterior to the antero-lateral view.

In addition to the muscle activity, there were other technical issues that affected the scanning process when attempting to obtain a complete 3D model from scanning the entire area. These technical considerations included:

a- Scanning the area in $360^{\circ}$ required a prolonged scanning time approximately twenty minutes. Prolonged scanning time is unwanted as the participant will move during the process and add extra motion artefact to the results. Minimizing the scanning time is recommended to reduce the motion artefact and increase the quality of the results. ${ }^{22,23}$ Moreover, the method will be applied in the future to injured participants, it would be unacceptable to expose wounds for extended period.

b- Scanning the area in $360^{\circ}$ generated many 3D reconstructed data around thirty-four in total. However, not all were used in the integration step. These data demanded time for manual noise cleaning, integration, and final processing. The overall processing time also was variable between the two scanning techniques.

c- Integration of different scans required drawing reference markers, during which time it was preferable to avoid touching the subject area.

In light of the above, scanning only one surface (required surface) of the body area in the stable manner was recommended as a standard 3D scanning technique as this technique eliminated the negative impact of the muscle activity and provided better image acquisition when compared to complete $360^{\circ}$ scanning technique.

\section{2-The ideal number of scans:}


Due to the absence of standards regarding the appropriate number of the scans and the number of

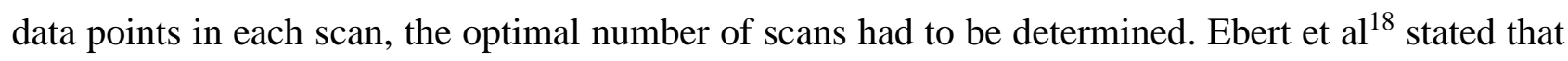
the number of scans is limited to two or three scans to avoid the negative effect of motion. Sansoni et $\mathrm{al}^{9}$ described that three scans are used to scan the forehead injuries. Ehlert et $\mathrm{al}^{35}$ considered that each scan has about 400,000 data points. However, ultimately the decision will be based on the type of used scanner, the aim of the application, the researcher needs and the scanned object details and size.

In order to decide roughly how many scans should be obtained by the Pico Scan in this work, the following test was performed. Different numbers of scans were taken to the wrist area. The anterior surface of the wrist joint was scanned with 2, 3, 4, 5, 6 scans respectively (Fig. 6). The volunteer was able to maintain stability during these scan's number. The presentation of final 3D images in the MeshLab software demonstrated that results were all similar to each other, all provided an acceptable appearance. However, when the wireframe 3D images on the screen were inspected more closely, differences were noticeable between images based on the number of scans. The wireframe 3D images resulting from more than three scans remained clear after zooming in, while the 3D images resulting from two or three scans lost their clarity upon closer inspection. This consideration is important since the presentation of forensic evidence in the courtroom usually requires on screen magnification and manipulation to display crucial findings. Therefore, the number of scans in the relevant view was decided to be more than three scans for wireframe mode presentation. However, in a practical field, it is expected that some scans will be affected by movement, the data that will be clearly affected by the movement artifact will not be processed; thus, the number of scans and the mode of presentation in the MeshLab software will be based mostly on researcher's decision.

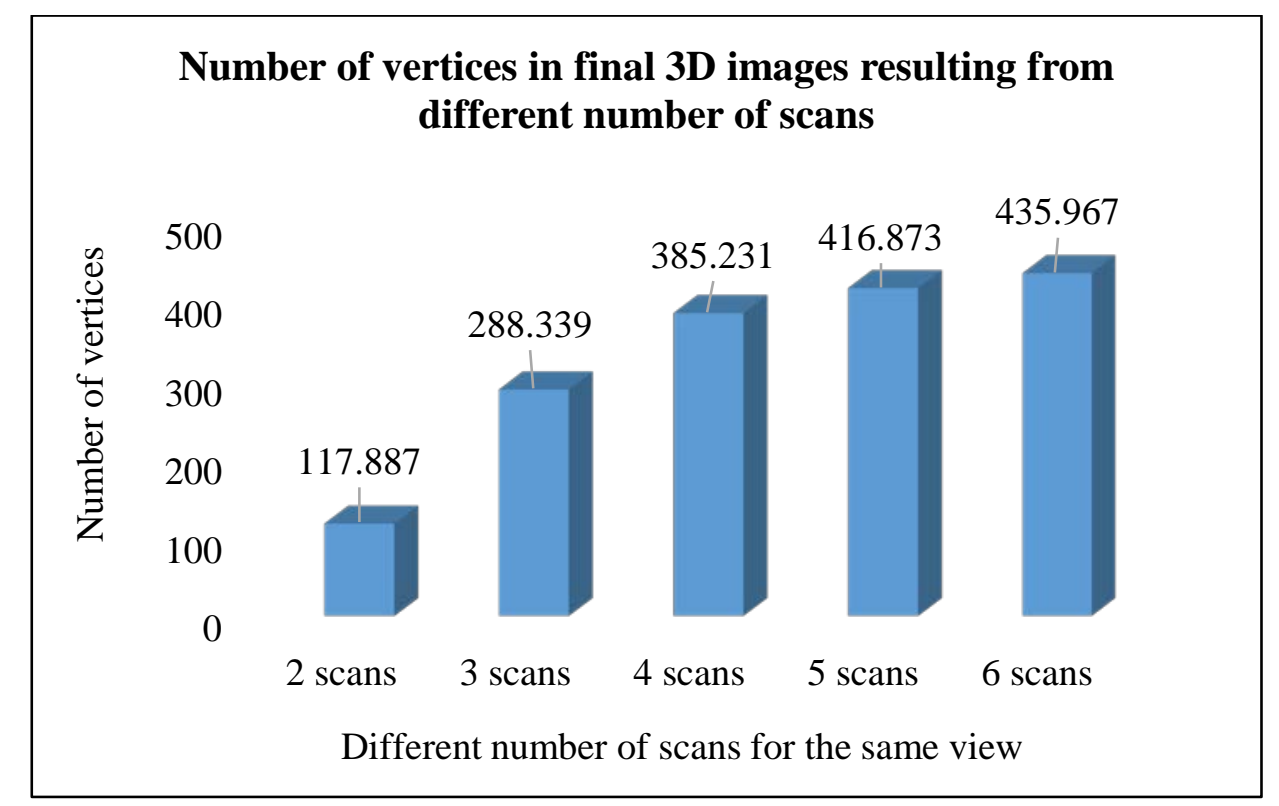

Fig. 6. More than three scans provide excellent number of vertices in the final 3D image.

3-Elimination of the environmental background noise in the acquired data: 
Although the maximum distance of the scanning process was determined, the Pico Scan 3D scanner was still able to record any object in the scanner's field of view beyond the maximum distance and generated a large amount of noise, back ground noise, in the data set. The formation of the noise was related to the value of the aperture and the intensity threshold of the light. When the aperture was larger (lower f/stop value) and the intensity threshold of the light was low, the background noise would be acquired clearly. Moreover, slow shutter speed generated the background noise even when the intensity threshold was high. Although the noise can be removed manually before the data processing, it is preferable to eliminate or reduce it from outset. This is because the camera sensor has limited range, acquiring noise during the scanning process will reduce the possibility of capturing more relevant data.

Therefore, a black background (screen) was placed behind the scanned area in order to eliminate the background noise formation in the acquired data set. The anterior aspect of the wrist joint was tested twice i.e. with and without the background. In each test, the area was scanned five times. Scanning was initiated under the same circumstances and using the same scanner parameters, such as calibration results, reconstruction and processing values. The total number of removed noise from all five-3D reconstructed raw data in the first test without using the black background was 669,397 points. While the total number of removed noise in the second test with using the background was 120,654 points. Therefore, using the black background reduced the noise formation from 669,397 to 120,654 points (Fig. 7). The eliminated noise was the background noise. It is worth mentioning that using the background did not only eliminate the background noise formation, but also provided the opportunity to the camera for more useful data acquisition. Therefore, using the black background was strongly recommended for scanning process.

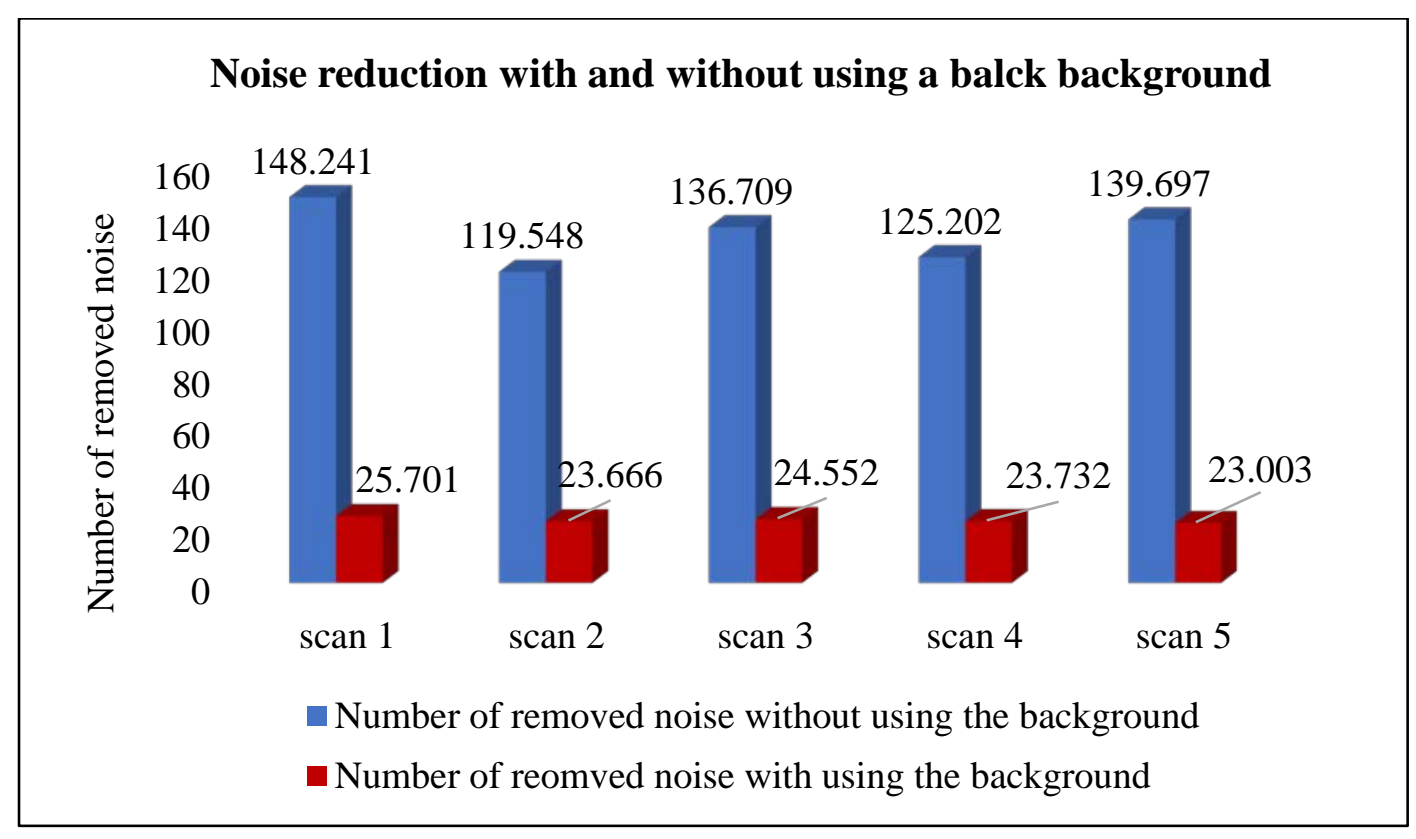

Fig.7. Using the black background eliminates the background noise. 


\section{4-Approaches to access different areas of the body:}

3D active surface scanning originally designed for static objects; thus, in order to enable the scanner accessing the different areas of the body, three different approaches were proposed, aimed to make the area easily accessible by facing the scanner. During these approaches, participants were aware to maintain the same position.

\section{Scanning approach No.1:}

It was proposed to access the different areas of the upper limp. The Pico Scan system was fixed on a tripod at a height about $110 \mathrm{~cm}$, the volunteers were in the standing position. A target surface of the upper limb was faced the scanner, rested on an ordinary stand matching the height of the scanner.

\section{Scanning approach No.2:}

It was proposed to access the different areas of the lower limp (foot, ankle and lower 2/3 of the leg). The Pico Scan system was fixed on the tripod at a height about $22 \mathrm{~cm}$, the volunteers were in the standing position. The wanted surface of the lower limb was faced the scanner. The upper third of the leg, knee joint and lower third of the thigh were accessed by facing the scanner while the tripod at a height about $55 \mathrm{~cm}$.

\section{Scanning approach No.3:}

It was proposed to access the torso (chest and abdomen). The Pico Scan system and the volunteers were in the same condition of the approach No.1. The area (the chest or abdomen) was faced towards the scanner, then the scanner level was adjusted to the chest or abdominal level.

Different areas of the upper, lower limb and torso were accessed by the previous suggested approaches. The scanner was able to create good quality of 3D images for different bare areas of upper and lower limbs with the exception of the anterior surface of the foot. Scanning result of this surface showed blurred visualization of the toes area in the Mesh Lab software, this may have occurred because the anterior surface of the foot was faced upward, it was not fully faced the scanner and not fully illuminated by the light, while scanning results of the medial and lateral surfaces of the foot had good visualization as these areas were faced and received the projected light equally.

Moreover, scanning results of the torso were unsatisfied as all reconstructed raw data had horizontal lines (Fig. 8) and distorted points (Fig. 9). The distorted points explained mostly by the movement of the volunteer despite trying to keep constant during scanning. While horizontal lines were resulting from thoracic and diaphragmatic breathing. Although, the PMP principle of the Pico Scan 3D scanner is characterized by high speed 3D measurement, the 3D reconstructed data were affected by the breathing as it is continuing physiological process. Asking the participant to stop breathing during the acquisition time should control the breathing artefacts, but will not be practical. However, scanning the torso area in the sitting position with a very cooperative volunteer or trying 
faster acquisition speed, less than 20 s/scan could overcome motion and breathing effects. Urbanova et $\mathrm{al}^{19}$ obtained the same result, they stated that scanning full body surface of a living person in the standing position by two types of 3D passive methods (VH1scanner and photogrammetry) are failed to produce satisfactory results due to the movement of a volunteer and breathing effect. While scanning the body in lying position influenced mainly by the breathing.

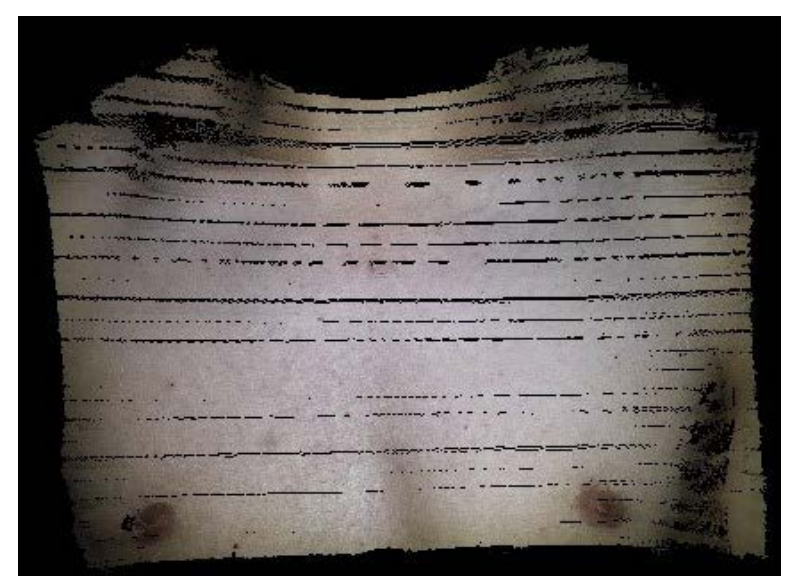

Fig. 8. 3D reconstructed raw data of the chest with multiple horizontal lines.

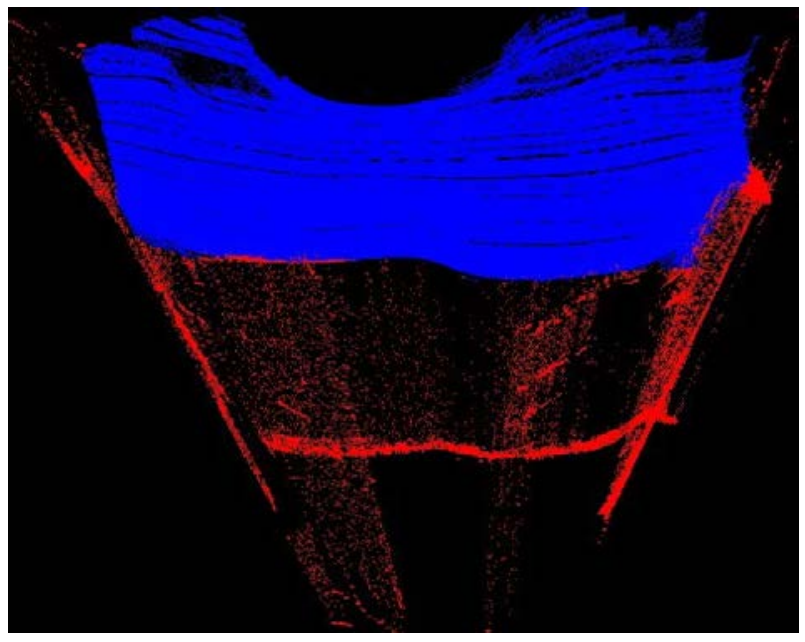

Fig. 9. 3D reconstructed raw data manipulated to show distorted points highlighted in red colour.

It is important to mention that the Pico Scan 3D scanner easily represented the moist surface, hairy areas, dark coloured skin and an area with coloured tattoo. While, Urbanova et $\mathrm{al}^{19}$ concluded that the algorithm of VH1 scanner skips forming a mesh and resulting a hole in hairy region while photogrammetry forms a model of distorted geometry for the same region. Also, the same methods are failed to generate satisfied results with moist area or area covered with body fluid. Villa ${ }^{22}$ cited that acquiring hairy areas by the passive 3D method requires special care as the camera of photogrammetric technique focus on hair rather than the injury, and scanning the wet area with water can cause error in photos orientation. Joun Tzou et $\mathrm{al}^{21}$ maintained that Vectra H1, Axisthree structured light scanner, DI3D, and 3dMD are 3D systems having limitations in scanning hairy areas. 
Traditional photography reduces 3D injuries to 2D level; moreover, it can be negatively affected by some practical and environmental factors, such as a distance, lighting condition and angel of acquisition. There are also additional factors to consider when applying new technology, such as level of expertise required to use the method, the cost of the equipment and the ease of interpretation. All of these constraints are also exhibited by passive and laser 3D surface technologies currently used in the forensic sciences. Nonetheless, structured light 3D surface digitization has been shown to be appropriate for recording injuries and has distinct advantages over traditional photography or photogrammetry, particularly with regard to accurate measurement. Unfortunately, much of the current application of this method is in the archaeological setting and as such there is no guidance for easily implementing the method on living individuals. This study analyzed the main 3D scanning issues using live test subjects and the Pico Scan 3D scanner to set up guiding principles for future use on injured victims. Guidance has been suggested based on the resultant gathered data. The study demonstrated that 1) scanning a required surface of the body area in the stable manner was more efficient when compared to complete $360^{\circ}$-scanning of an area because the particular surface scanning technique eliminated the negative impact of the muscle activity and provided better image acquisition; thus, it used as a standard 3D scanning technique to reconstruct bare different areas of the live subject. 2) More than three scans were ideal to obtain optimum wire frame mode representation of the scanned view; however, the number of scans and the mode of presentation will be based mostly on researcher's decision. 3) Using a black background was highly recommended to eliminate the background noise in acquired raw data. 4) Three approaches were suggested to access the different areas of the body. Although, 3D scanning of live subjects using the Pico scan 3D scanner faced some challenges, the scanner was beneficial in generating tesxtured-3D models of different bare areas of upper and lower limb and will be promising to reconstruct injuries from these areas. Therefore, a further work will be conducted in a practical setting to reconstruct different types of forensic injuries from wounded victims, so that the effectiveness of this method can be evaluated in the field.

\section{References}

1. Thali MJ, Braun M, Brueschweiler W, Dirnhofer R. Morphology imprint' determination of the injury-causing weapon from the wound morphology using forensic 3D/ CAD- suspected photogrammetry. Forensic Science International Journal. 2003; 132:177-18. doi: http://dx.doi.org/10.1016/S0379-0738(03)00021-5.

2. Krouskop TA, Baker R, Wilson MS. A noncontact wound measurement system. Journal of Rehabilitation Research and Development. 2002; 39: 337-346. 
3.Thali MJ, Braun M, Dirnhofer R. Optical 3D surface digitizing in forensic medicine: 3D documentation of skin and bone injuries. Forensic Science International Journal. 2003; 137: 203208. doi: 10.1016/j.forsciint.2003.07.009.

4. Casas L, Castaneda B, Treuillet S. Imaging technology applied to chronic wounds: a survey. Proceedings of the 4th International Symposium on Applied Sciences in Biomedical and Communication Technologies. Oct 2011, Barcelona, Spain, pp.1-5. doi: 10.1145/2093698.2093865.

5. Thali MJ, Braun M, Markwalder Th H, Brueschweiler W, Zollinger U, Malik NJ, Yen K, Dirnhofer R. Bite mark documentation and analysis: the forensic 3D/CAD supported photogrammetry approach. Forensic Science International Journal. 2003; 135:115-121. doi: 10.1016/S03790738(03)00205-6.

6. Evans S, Baylis S, Carabott R, Jones M, Kelson Z, Marsh N, Payne-James J, Ramadani J, Vanezis P , Kemp A. Guidelines for photography of cutaneous marks and injuries: a multi-professional perspective. Journal of Visual Communication in Medicine. 2014: 37: 3-12. doi: 10.3109/17453054.2014.911152.

7. Boyat AK, Joshi BK. A review paper: noise models in digital image processing. Signal and Image Processing: An International Journal (SIPIJ). 2015: 63- 75. doi: 10.5121/sipij.2015.6206.

8. Bruschweiler W, Braun M, Dirnhofer R, Thali MJ. Analysis of patterned injuries and injury-causing instruments with forensic 3D/CAD supported photogrammetry (FPHG): an instruction manual for the documentation processes. Forensic Science International Journal. 2003; 132: 130-138. doi:10.1016/S0379-0738(03)00006-9.

9. Sansoni G, Cattaneo C, Trebeschi M, Gibelli D, Porta D, Picozzi M. Feasibility of contactless 3D optical measurement for the analysis of bone and soft tissue lesions: new technologies and perspectives in forensic sciences. Journal of Forensic Science. 2009; 54: 540-545. doi: 10.1111/j.1556-4029.2009. 01041.x.

10. De Sainte Croix MM, Gauld D, Forgie AH, Lowe R. Three- dimensional imaging of human cutaneous forearm bite marks in human volunteers over a 4-day period. Journal of Forensic and Legal Medicine. 2016; 40: 34-39. doi: 10.1016/j.jflm.2016.02.003.

11. Geng J. Structured-light 3D surface imaging: a tutorial, Advances in Optics and Photonics Journal. 2011; 3: 128-160. doi: 10.1364/AOP.3.000128.

12. Salvi J, Fernandez S, Pribanic T, Llado X. A state of the art in structured light patterns for surface profilometry. Pattern Recognition Journal. 2010; 43: 2666-2680. doi: 10.1016/j.patcog.2010.03.004. 13. Errickson D, Thompson TJU, Rankin BWJ. The application of 3D visualization of osteological trauma for the courtroom: A critical review, Journal of Forensic Pathology and Imaging. 2014; 2: 132137. doi: 10.1016/j.jofri.2014.04.002. 
14. Subke J, Wehner HD, Wehner F, Szczepaniak S. Streifenlichttopometrie (SLT): a new method for three-dimensional photorealistic forensic documentation in colour. Forensic Science International. 2000; 113: 289-295. doi: 10.1016/S0379-0738(00)00236-X.

15. Akca D. 3D modelling of cultural heritage objects with a structure light system. 2012; 12:139152.

16. McPherron SP, Gernat T, Hublin J. Structure light scanning for high-resolution documentation of in situ archaeological finds. 2009; 36: 19-24. doi: 10.1016/j.jas.2008.06.028.

17. Thali MJ, Braun M, Buck U, Aghayev E, Jackowski C, Vock P, Sonnenschein M, Dirnhofer R. Virtopsy-scientific documentation, reconstruction and animation in forensic: individual and real 3D data based geometric approach including optical body/object surface and radiological CT/MRI scanning. Journal Forensic Science. 2005; 50: 428-442. doi: 10.1520/JFS2004290.

18. Ebert LC, Flach P, Schweitzer W, Leipner A, Kottner S, Gascho D, Thali MJ, Breitbeck R. Forensic 3D surface documentation at the Institute of Forensic Medicine in Zurich-workflow and communication pipeline. Journal of Forensic Radiology and Imaging. 2015; 5: 1-7. doi: 10.1016/j.jofri.2015.11.007.

19. Urbanova P, Hejna P, Jurda M. Testing photogrammetry-based techniques for three-dimensional surface documentation in forensic pathology. Forensic Science International Journal. 2015; 250: 7786. doi: http://dx.doi.org/10.1016/j.forsciint.2015.03.005.

20. Lohry WF, Chen V, Zhang S. Absolute three-dimensional shape measurement using coded fringe patterns without phase unwrapping or projector calibration. Optics Express Journal. 2014; 22: 12871301. doi: 10.1364/OE.22.001287.

21. John Tzou C, Artner NM, Pona I, Hold A, Placheta E, Kropatsch WG, Frey M. Comparison of three-dimensional surface-imaging systems. Journal of Plastic, Reconstructive and Aesthetic Surgery. 2014; 67: 489-497. doi: 10.1016/j.bjps.2014.01.003.

22. Villa C. Forensic 3D documentation of skin injuries. International Journal of Legal Medicine. 2016. doi: 10.1007/s00414-016-1499-9.

23. Kovacs L, Zimmermann A, Brockmann G, Gühring M, Baurecht H, Papadopulos NA, Schwenzer-Zimmerer K, Sader R, Biemer E, Zeilhofer HF. Three-dimensional recording of the human face with a 3D laser scanner. Journal of Plastic, Reconstructive \& Aesthetic Surgery. 2006; 59: 1193-1202.

24. Yalla VG, Hassebrook LG. Very high resolutions 3D surface scanning using multi-frequency phase measuring profilometry. Proceedings of SPIE 5798, Spaceborne Sensors II. 2005. doi: $10.1117 / 12.603832$. 
25. Wang Y, Liu K, Hao Qi, Lau D, Hassebrook L. Multicamera Phase Measuring Profilometry for Accurate Depth Measurement. Proceedings of SPIE 6555, Sensors and Systems for Space Applications 655509. 2007. doi: 10.1117/12.720068.

26. Ishii I. A Coded Structured Light Projection Method for High-Frame-Rate 3D Image Acquisition. http://cdn.intechopen.com/pdfs-wm/32185.pdf; 2012 Accessed 27.10. 2016.

27. Eiríkssona ER, Wilma J, Pedersenb DB, Aanæsa H. Precision and accuracy parameters in structured light 3D scanning, ISPRS-The International Archives of the Photogrammetry, Remote Sensing and Spatial Information Sciences Volume XL-5/W8: 7-15. 2016. doi:10.5194/isprsarchivesXL-5-W8-7-2016.

28. Georgopoulos A, Ioannidis $\mathrm{CH}$, Valanis A. Assessing the performance of a structured light scanner. International Archives of Photogrammetry, Remote Sensing and Spatial Information Sciences, Vol. XXXVIII, Part 5, Commission V Symposium, Newcastle upon Tyne, UK. 2010.

29. Salvi J, Pages J, Batlle J. Pattern codification strategy in structured light systems, Pattern Recognition Journal. 2004; 37: 827-849. doi: 10.1016/j.patcog.2003.10.002.

30. Corsarca C, Jocea A, Savu A. Analysis of error sources in Terrestrial Laser Scanning. http://www.uab.ro/geocad/upload/18_144_Paper11_RevCAD09_2009.pdf; 2009 Accessed 24. 6. 2016.

31. Errickson D, Thompson T, Rankin B. An Optimum Guide for the Reduction of Noise using a Surface Scanner for Digitising Human Osteological Remains. http://www. guides.archaeologydataservice.ac.uk/g2gp/CS_StructuredLight; 2015 Accessed 20. 9. 2016.

32. Gorthi SS, Rastogi P. Fringe projection techniques. Optics and Laser in Engineering Journal. 2010; 48: 133-140. doi: 10.1016/j.optlaseng.2009.09.001.

33. Huang PS, Zhang C, Chiang FP. High-speed 3-D shape measurement based on digital fringe projection. Optical Engineering. 2003; 42:163-168. doi:10.1117/1.1525272.

34. Thali MJ, Braun M, Brueschweiler W, Dirnhofer R. Matching tire tracks on the head using forensic photogrammetry. Forensic Science International Journal. 2000; 113: 281-287.doi: 10.1016/S0379-0738(00)00234-6.

35. Ehlert A, Salah Z, Bartz D. Data reconstruction and visualization techniques for forensic pathology. Proceedings of the Eighth Joint Eurographics / IEEE VGTC Conference on Visualization. 2006. doi:10.2312/VisSym/EuroVis06/323-330. 